\title{
The Research of Influenza H1N1's Transmission Based on Artificial Society
}

\author{
Song Zhi Chao, Ge Yuan Zheng, Duan Hong, Meng Rong qing, and Qiu Xiao Gang
}

\begin{abstract}
As the computer technology develops rapidly, the Artificial Society Method has been applied in lots of fields, which uses computers to build one virtual laboratory and studies the questions in it by experiments. This paper researches the transmission of influenza H1N1 which spreads globally. In this paper we construct a simple artificial village by building Agents and the hierarchical contact relationships among them firstly. Then activity rules of Agents are designed and the SEIR model is applied in the influenza H1N1's transmission experiments. At last parameters and rules are set. How the initial number of the infected and the degree of the initial infected person in the contact network affect the transmission of the influenza H1N1 have been studied by two simulation experiments. The results show that the transmission speed and the number of the infected in experiments are positively correlated with the number of the initial infected and the degree of the initial infected person in the contact network.
\end{abstract}

Index Terms-Artificial society METHOD, influenza H1N1, SEIR, contact relationships.

\section{INTRODUCTION}

As we all know infectious diseases have always threatened human beings' lives and killed so many people in history. The Plague of Justinian killed almost half of the Europeans in A. D. 600; in the sixteenth century the smallpox flooded in Europe and the death toll reached ten percent of the population in Europe at that time; and in the eighteenth century the bubonic plague emerged everywhere in Europe and brought infinite pain to human beings. Nowadays, though sanitation facility has been improved so much and the developments of medicine science have reduced the threat of infectious diseases, in recent years new infectious diseases, such as SARS and influenza H1N1, have still killed many people and made the global society scared. Therefore researches on how to eliminate or reduce the harm from infectious diseases are still very important.

Fundamentally, the most valuable research on controlling the transmission of the infectious diseases should be medicine technology or bacterin. However, this process always entails a long time, for example the vaccinia cure for smallpox was found by Edward Jenner in the eighteenth century hundreds of years after the smallpox appeared. In fact, medicine scientists have not found all the methods to cure different infectious diseases. What's more, it is so difficult to find a method to cure new infectious diseases such as SARS

Manuscript received February 1, 2014; revised April 4, 2014. This work was supported in part by National Nature and Science Foundation of China under Grant No. 91024030.

The authors are with College of Information System and Management, National University of Defense Technology, Changsha, China (e-mail: song_zhichao@139.com). or influenza H1N1 in a short time. So our need cannot be met only by the therapy study to control the infectious diseases spread. According to the analysis we find that strong infectiousness is also a very important reason why infectious diseases are so devastating except their high mortality rate. Thus more and more researchers have focused on the transmission of the diseases. They hope that they can reduce the threat by controlling the spread of diseases when the transmission rules are known.

Researches based on mathematical models have acquired lots of achievements [1]-[3] but such methods have some shortages. Most of the infectious diseases transmission models are based on the differential dynamics. The computing is always very complex and solutions of the differential functions are extremely sensitive to initial conditions. As the differential dynamics are mainly used to compute the transmission results, random factors cannot be considered and the transmission process under different conditions cannot be observed.

More and more researchers wish that they could do some experiments so that they can observe infectious diseases transmitting among the crowds under different kinds of conditions more deeply. Nevertheless, this kind of experiments cannot be done in our real life because they are so inhuman. That is why the researchers prefer studying the disease transmission by mathematical models in the past. As the high-speed development of computer technology, the idea can be realized. We can use computers to build a virtual infectious diseases transmission laboratory. Thus researchers can take lots of simulation experiments about the diseases transmission and learn more about it in the laboratory.

This paper studies how influenza H1N1 spreads among the crowds. Based on the Artificial Society Method [4] we use the Agent modeling technology to build one artificial village with 10000 persons and the social contact relationships among Agents are also constructed according to the complex network theory. Then the influenza transmission simulation among the crowds is realized. The remainder of this paper is organized as follows. In Section II we introduce the influenza H1N1. How to build the artificial society to research influenza H1N1 transmission is introduced in Section III. Section IV gives the SEIR model. In Section V we show the construction process of the artificial village. Section VI describes two simulation experiments we have done and the conclusions are given in Section VII.

\section{INFLUENZA H1N1}

In 2009 influenza H1N1 broke out in Mexico and spread around the world quickly. According to the statistics, the global death toll caused by influenza H1N1 exceeded 6200. 
In China, influenza H1N1 was also quite devastating. According to the statistics from Chinese Ministry of Health, the infected have reached 92904 and 200 infected persons were dead of it until December 7, 2009.

Medicine researches on influenza H1N1 have shown that it is similar to the common flu. The disease spreads around crowds when they contact each other by the air. When a person is infected, symptoms usually do not appear very soon and there is a latent period. The symptoms come out after this latent period passes. Once the infected person is cured, he/she will have immunity to this disease. As different from the common flu, the infectivity and mortality rate of influenza H1N1 are much higher. So we can apply the SEIR model [5] which considers the latent period in the infection spread simulation experiments.

\section{CONSTRUCTION OF ARTIFICIAL SOCIETY}

\section{A. Construction Design of Artificial Society}

The Artificial Society Method [4] is proposed by the Rand Company to research the effect on the society from the information technology. This method is to bring a virtual laboratory for researchers to study the social questions by the means of taking experiments. The main idea of the Artificial Society Method is to simulate individuals in our human being society. Then the computing experiments can be taken in the computer using these artificial individual models and some interesting phenomena might emerge by their interacting with each other. Thus we can use this method to research the infectious diseases transmission. If we can build the models for infectious diseases and make them run in the artificial society, the transmission of infectious disease under different conditions can be observed.

Our human being society is made up with large number of individuals. There are so many complex social relations among them. Every individual acts according to his/her own mind. Being an abstract of the real society, the artificial society can be constructed by building large numbers of models for the individuals and making them correlate with each other according to some rules.

For the research of infectious diseases transmission the most important element leading to such diseases spreading around the crowds is the contact behavior between the infected and others. Intuitively it seems that whether a contact behavior takes place or not is directly related with the space between two persons but we can also find that the persons we contact in our life always have some relationships with ourselves. For example the possibility that we contact with our chums must be much higher than the strangers. Therefore the social relationships among human beings decide whether contact behavior can take place among them or not. In this paper, we call the relationships leading to the infectious diseases spreading contact relationships and the artificial society is made up with individual models and the contact relationships among them. The individual models' activity is the contact behaviors that may lead to the infectious diseases spreading. Whether the contact behaviors take place is decided by the contact relationships.

\section{B. Agent Model Design}

The individual models in the artificial society are realized by the Agent modeling technology [6]. The Agent model design of the artificial society is shown in Fig. 1. Every Agent has two parts: the relationship list and its own property list. The relationship list comprises three lists: a strong tie list, an ordinary tie list and a weak tie list. All kinds of relationship lists of Agent i comprise three parts: IDs of the Agents that Agent i may contact with, their contact number of times, and their contact possibilities. The property list has five parts: 1) ID of the Agent itself; 2) infected persons' states, including susceptible, latent, symptomatic, accepting treatment and immune; 3 ) the latent period; 4) the symptom emerge period; $5)$ the accepting treat period.

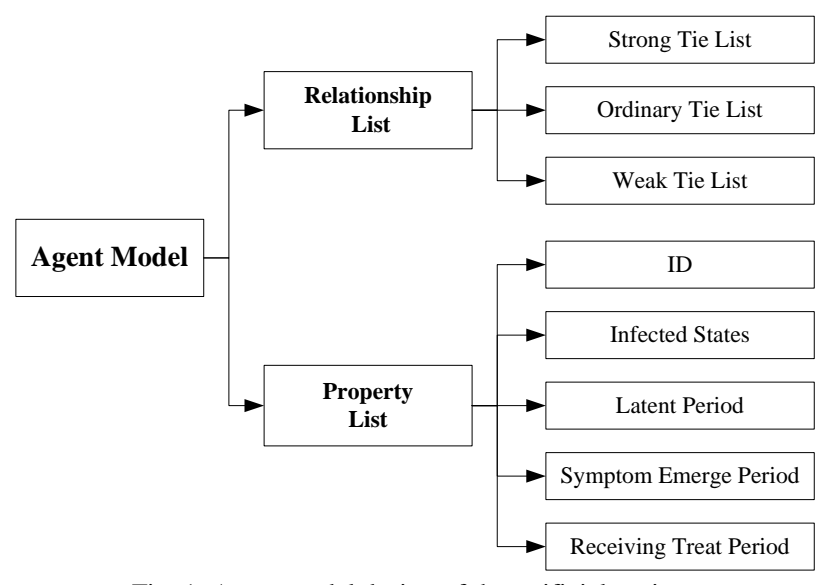

Fig. 1. Agent model design of the artificial society.

\section{Construction of the Contact Relationships}

In this paper we divide the contact relationships into three layers: strong ties, ordinary ties and weak ties [7]. The strong ties describe the relationships leading to the contact behaviors among human beings that take place quite often. The families, classmates, good friends and so on belong to this kind of relations. In the simulation an Agent almost contacts with another Agent which has a strong tie with it in every simulation step. Relationships that the ordinary ties describe do not happen so that often as the strong ties. This kind of relationships always exists among neighbors. The weak ties are used to describe the relationships that lead to the contact behaviors take place by accident.

Strong ties include family relationships, good friendship, relationships between teacher and students etc. Such relationships like family relationships are usually very stable and have some special mode. If we use the complex network theory to describe it, we can use the regular networks like globally coupled network in which every pair of nodes has the same kind of relationship. Nevertheless, the good friendship cannot be described like that because the relationships such as friendship do not have such a stable mode and is more complex. Thus we construct the relationships according to the idea that randomly choosing other Agents to be its friends by a possibility $\mathrm{P}_{\mathrm{i}}$, which is proposed by Newman and Watts [8] to build the small-world networks. Then the construction of strong ties would include two steps. The first step is to build the relationships that have special mode we have known by the regular networks. The second is to choose the other relations like their friends at random.

The ordinary ties are built by the contact simulation [9]. 
The main idea is to get the ordinary ties among Agents according to their contact number of times in the simulation. Our life experiences tell us that if one person has a big family or has lots of good friends he/she can contact with more other people except his/her family members and good friends, and the possibility of contact behaviors happen will be very high. It is similar that if the social clique of one person is small the possibility of contacting with others out of his clique is much smaller. What's more, if the contact behavior happens once between two persons, there may be some relationship between them and they may contact with each other again. Thus the possibility of contact behaviors taking place should be increased as the contact number of times increases.

Therefore the contact possibility among Agents in the contact simulation is designed as (1):

$$
P_{i j}=\left(N_{i}+N_{j}\right) /(2 N)+n_{i j} / T \text {, if } P_{i j}>1 \text {, then } P_{i j}=1 \text {. }
$$

Here we assume that Agent $i$ and Agent $\mathrm{j}$ contact with each other. The contact possibility is $P_{i j}$. $N_{i}$ and $N_{j}$ is their own numbers of strong ties respectively. $N$ is the total number of Agents. $n_{i j}$ is the contact number of times between them. $T$ is the total number of times the simulation runs.

The detailed building process is as follows.

1) An ordinary tie list is built for every Agent. The Agents' IDs except themselves are all put into the list and the contact number of times is 0 .

2) The work will be done for all the Agents as follows. At first the contact possibility $P_{i j}$ of all the Agents in the ordinary tie list of Agent $i$ will be computed. Then whether the contact behaviors take place among the Agents and Agent $i$ will be judged. At last if the contact behavior between two Agents happens, the contact number of times $n_{i j}$ will be updated for the two Agents.

3) Step 2 will be run $T$ times.

4) The Agents' IDs in the list will be deleted if their contact number of times is less than the threshold $\mathrm{c}$. Thus ordinary ties of Agents will be completed and saved in their ordinary tie lists.

The weak ties lead to the accidental contact behaviors. Thus we assume that the weak ties exist among one Agent $i$ and all the other Agents out of its strong tie list and ordinary tie list. In the infectious disease spread simulation some Agents in the weak tie list of Agent $i$ will be chosen at every simulation step to be the accidental contact objects.

\section{SEIR MODEL}

In 1927 Kermack and Mckendrick [3] divided population into three kinds: the susceptible, the infected and the recovered to research the bubonic plague spread in London and the plague transmitted in Bombay. He used S, I, R to represent the numbers of the three kinds of people and built one differential dynamics model. The model was called SIR model. Then the SIR model had been developed so much and the SEIR model [5] is one of them which can describe the infectious diseases transmission with a latent period. The main idea is to divide the population into four kinds. The persons never infected are called the susceptible. The infected persons in the latent period are called the latent. The infected persons in the symptom emerge period can be called the symptom emerge persons or the infected. The person who has been cured can be called the recovered.

According to this idea, a person who is infected by influenza H1N1 will be through the process as shown in Fig. 2.

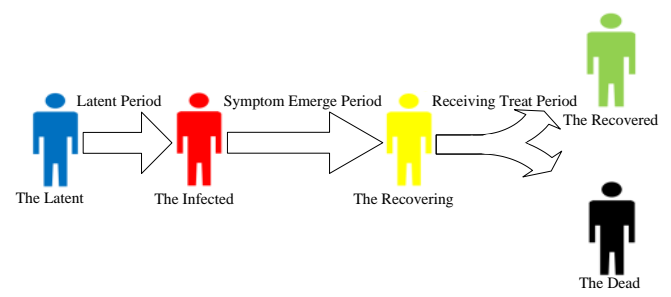

Fig. 2. States transforming of a person infected by influenza H1N1.

\section{REALIZATION OF THE ARTIFICIAL VILLAGE}

To research the influenza H1N1 transmission around the crowds this paper built one artificial village which has 10000 Agents by MATLAB according to the distribution (Fig. 3) of the households in the village from the sixth national demographic census of Beijing in 2010.

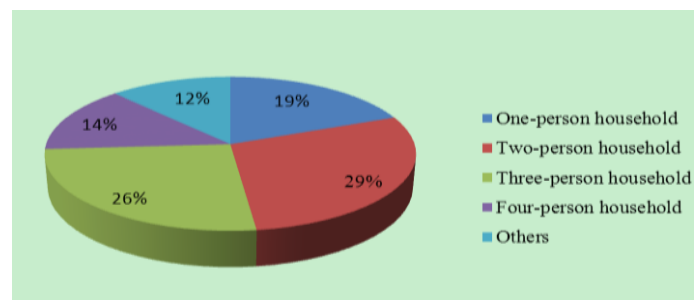

Fig. 3. Mean distribution of households in the villages of Beijing.

To construct the artificial society more simply the 10000 Agents are divided into many households according to the distribution. The setting is as the Table I lists below in which One-P-H stands for One-person-household.

TABLE I: HOUSEHOLDS SETTING OF THE ARTIFICIAL VILLAGE

\begin{tabular}{lcccccc}
\hline \hline & One & Two & Three & Four & Five & Six \\
& -P-H & -P-H & $-\mathrm{P}-\mathrm{H}$ & $-\mathrm{P}-\mathrm{H}$ & $-\mathrm{P}-\mathrm{H}$ & $-\mathrm{P}-\mathrm{H}$ \\
\hline Population number & 1870 & 2900 & 2640 & 1420 & 690 & 480 \\
Household number & 1870 & 1450 & 880 & 335 & 138 & 80 \\
\hline \hline
\end{tabular}

The process of building the artificial village is as follows.

1) At first the 10000 Agents models are built using the Agent technology and the parameters are initialized. The ID of every Agent is given. The states of all the Agents are set as the susceptible. The other parameters and social relationships are blank.

2) Then the social relationship lists are completed as we have introduced in Section III. The strong ties are constructed by two steps. Firstly the family relationships are built. Everyone in a family has strong ties with the others and it can be described by the globally coupled network. Thus we divide all the Agents into lots of groups according to the households setting. The social relationships are recorded in the strong tie lists. Secondly the good friends or colleagues of the Agents are chosen randomly based on the possibility $P_{i}=0.0004$. The ordinary ties are built by the contact simulation 
experiment method. The number of times the simulation runs $\mathrm{T}$ is set by 500 . The threshold $c=25$ and the ordinary tie lists are completed in this process.

To understand the contact relationships more clearly we choose 5-10 Agents randomly to build the weak tie lists as we have introduced in Section III. If an Agent is regarded as a node and the relationships which comprises strong ties, ordinary ties and weak ties are regarded as edges a contact relationship network is built. The structure of the network is shown in Fig. 4 and the degree distribution of the network is shown in Fig. 5.

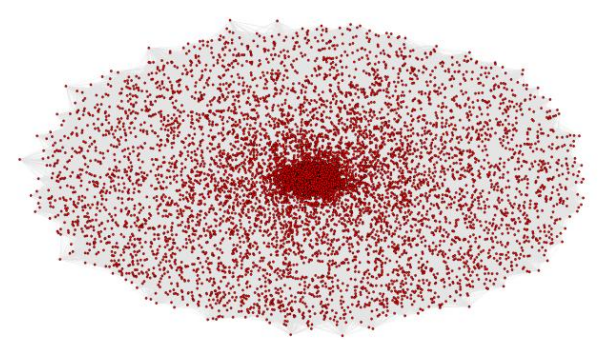

Fig. 4. The structure of the contact relationship network.

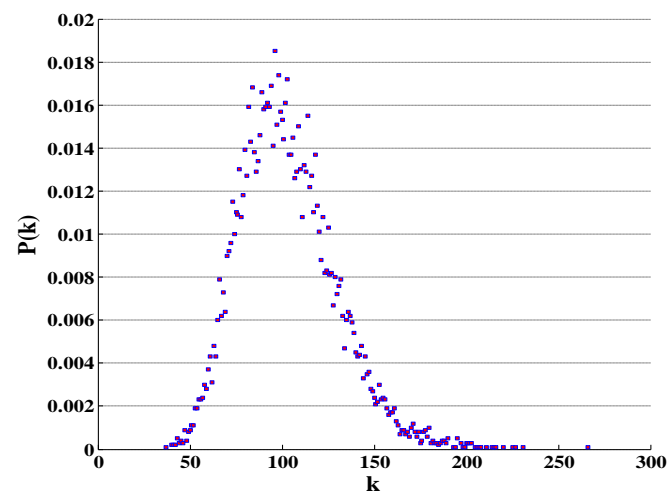

Fig. 5. The degree distribution of the contact relationship network.

\section{THE INFLUENZA H1N1 TRANSMISSION SIMULATION EXPERIMENTS}

The infectious diseases transmission model is defined as follows.

1) The simulation step is set by day.

2) The latent period time follows the uniform distribution $U$ [2], [4] [10], and the symptom emerge period time follows the uniform distribution $U$ [1], [3].

3) When the infected Agent is in the latent period the infectious disease transmission possibility is 0.05 . When the infected Agent is in the symptom emerge period time, the possibility is 0.11 [11]. The infected persons will stay in the hospitals when they are in the accepting treatment period. Therefore, we assume that the infected persons cannot infect others during this period.

The activities rules are as follows. It is for the Agents except those in the accepting treatment period.

1) The Agent contacts the others which have strong ties with it at the possibility 0.8 .

2) The Agent contacts the others which have ordinary ties with it according to the possibilities recorded in the ordinary tie list.

3) The Agents contact the other Agents in the weak tie list by choosing 5-10 Agents in their weak tie lists randomly.

\section{A. Simulation Experiment I}

For influenza $\mathrm{H} 1 \mathrm{~N} 1$, the spread in a district is always caused by the infected persons outside. The number of initial infected people may affect the transmission of influenza H1N1. In this paper we have researched the influenza H1N1's transmission under different numbers of people infected by the disease in the beginning. In the experiment we chose 1, 3 and 10 Agents to be the initially infected. The experiment was run for 20 times. Then the number of newly infected people and the accumulative number of infected people every day under the condition that different numbers of initially infected people are shown in Fig. 6 and Fig. 7.

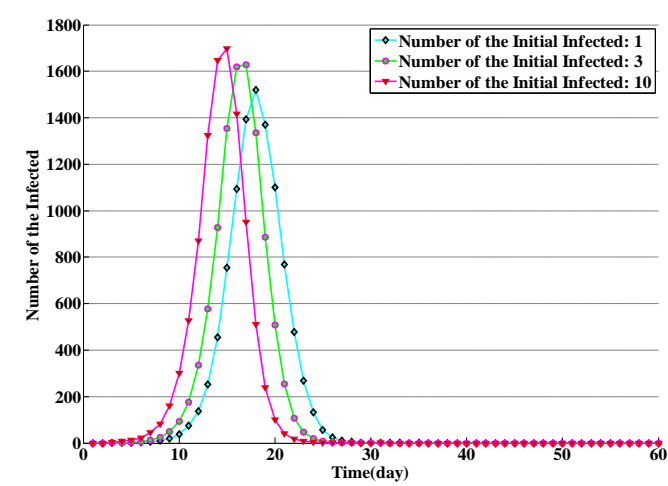

Fig. 6. The number of newly infected people.

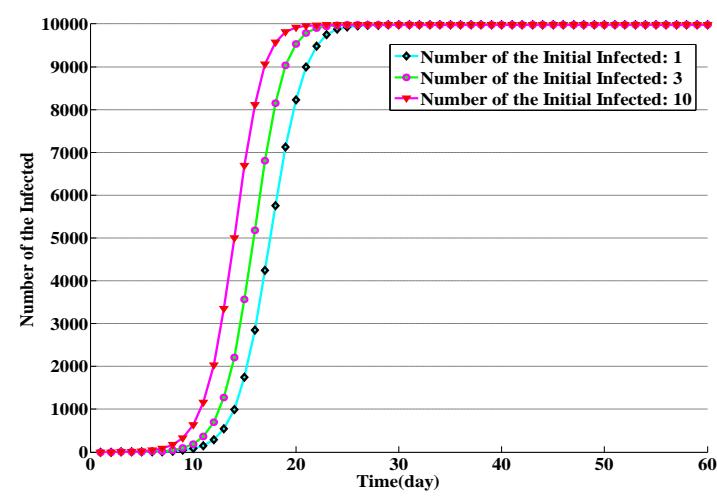

Fig. 7. The accumulative number of infected people.

From the figures we can find that if human beings do not change their activities during the transmission of influenza H1N1 no matter the number of the initially infected people is 1,3 or 10 , almost all the people will be infected. The difference is the transmission speed and the infected peak value. If the number of the initially infected people is 1 , the infection begins to spread heavily at the tenth day and the infected peak value is 1500 at the eighteenth day. When the number of the initially infected people is 3 and 10 the infection begins to spread heavily at the seventh day and the ninth day, the infected peak value is 1600 and 1700 at the seventeenth day and fifteenth day respectively. Thus we can have a conclusion that the infection will spread more heavily if the number of the initial infected is more.

\section{B. Simulation Experiment II}

If one person always contacts with others or he/she has lots of friends he/she may infect more people once he/she is infected by influenza H1N1. So we have taken an experiment 
to observe the transmission of this disease when an initially infected person has different numbers of friends. When the relationships among the Agents are regarded as a network the degrees of Agents can describe how many friends an Agent has because the degree of a node in the complex network means the number of nodes directly link to it. In the experiment we chose five Agents whose degrees are 35, 84, 150,206 and 257 to be the initial infected separately to start the transmission simulation. The experiment was also run 20 times. The number of newly infected people and the accumulative number of infected people everyday are recorded in Fig. 8 and Fig. 9.

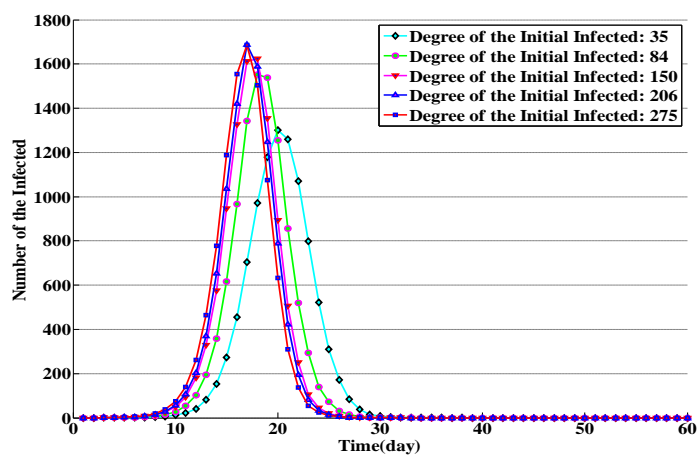

Fig. 8. The number of newly infected people.

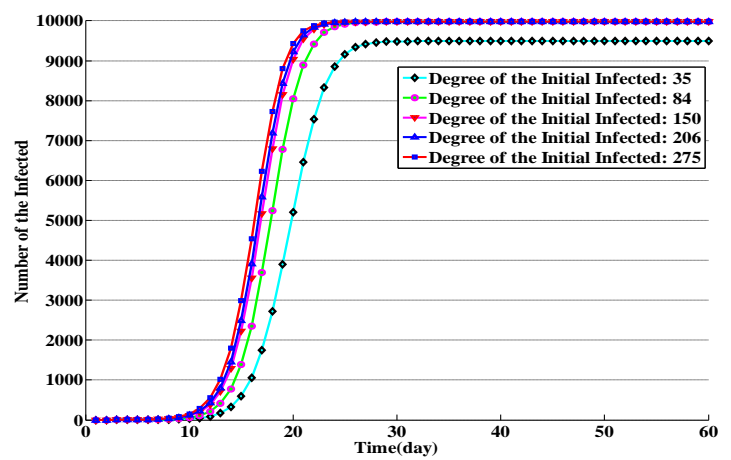

Fig. 9. The accumulative number of infected people.

The figures above show us that influenza H1N1 spreads more heavily as the degree of the initial infected person in the contact network increases. This phenomenon can be seen from the speeds of the newly infected increasing and the infected peak values. For example when the degree of the initially infected people is 35 the rising speed of the curve is slower than the others. For the current settings in this simulation experiment we find that if the degree of the initial infected exceed some threshold all people will be infected. For example, when the degree of the initially infected people is 35 there are 9400 Agents infected but almost all the people are infected under the other conditions. That is because the small degree lead to the decrease of the influenza H1N1's transmission speed and the infected Agents cannot infect so many other Agents before they go to hospital for treatment.

\section{CONCLUSION}

In this paper, a simple artificial village with 10000 Agents is built by constructing the contact relationships among Agents and designing the activities rules. According to the Artificial Society Method we have conducted two simulation experiments about influenza H1N1's transmission in the artificial village base on the SEIR model. We find that the influenza H1N1's transmission speed and the number of the infected in the experiments are positively correlated with the number of the initially infected people and the degree of the initial infected people in the contact network. Therefore, in the beginning of influenza H1N1's spreading, if the infected persons especially those having many friends can be treated very well in the hospitals the transmission can be greatly curbed. In this paper we just introduced a simple idea to research the transmission of infectious diseases with the help of Artificial Society Method. We will do more researches in two directions in the future. Firstly, the structure of the social networks leading to influenza H1N1 spreading around the crowds will be continue to study and the algorithm to construct the contact relationships will be improved to make it more exact. Secondly, as influenza H1N1 can be spread by the air, we should consider the environment and research how to build the environment models in the artificial society.

\section{REFERENCES}

[1] W. H. Hamer, Epidemic Disease in England: The Evidence of Variability and of Persistency of Type, Harvard: Bedford Press, 1960.

[2] R. Ross, The Prevention of Malaria, John Murray, Albemarle Street, California, USA, 1911.

[3] W. O. Kermack and A. G. McKendrick, "Contributions to the mathematical theory of epidemics," The Royal Society of London: Series A, vol. 115, pp. 700-721, 1927.

[4] C. H. Builder and S. C. Banks, Artificial Societies: A Concept for Basic Research on the Societal Impacts of Information Technology, Santa Monica, CA, RAND, USA, 1991.

[5] M. Y. Li and J. S. Muldowney, "Global stability for the SEIR model in epidemiology," Mathematical Bioscience, vol. 125, no. 2, pp. 155-164, February 1995.

[6] R. Sun, Cognition and Multi-Agent Interactions: From Cognitive Modeling to Social Simulation, Cambridge University, New York, USA, 2006.

[7] M. Granovetter, "The strength of weak ties," American Journal of Sociology, vol. 78, no. 6, pp. 1360-1380, May 1973.

[8] M. E. J. Newman and D. J. Watts, "Scaling and percolation in the small-world network model," Physical Review E, vol. 60, no. 6, pp. 7332-7342, May 1999

[9] H. M. Singer, I. Singer, and H. J. Herrmann, "Agent-based model for friendship in social networks," Physical Review E, vol. 80, no. 2, pp. 026113, August 2009

[10] L. Q. Zhang, "Epidemiological and clinical features of influenza A (H1N1) 2009 in Pinggu district, Beijing," Capital Journal of Public Health, no. 4, pp. 162-165, August 2010.

[11] Y. Z. Ge, Z. C. Song, X. G. Qiu, H. Song, and Y. Wang. "Modular and hierarchical structure of social contact networks," Physica A: Statistical Mechanics and its Applications, vol. 392, no. 19, pp. 4619-4628, October 2013.

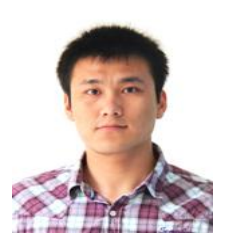

Song Zhichao is currently a $\mathrm{PhD}$ student at research center of military computational experiments and parallel system technology, National University of Defense Technology, Changsha, China. His research interests focus on complex system modeling and social networks analysis.

He has joined a project supported by National Nature and Science Foundation of China under Grant No. 9102403 since 2010 . His job is to build the artificial society in which lots of experiments in the society fields can be done. In his job he cares more about the artificial society building methods and the social networks modeling. 